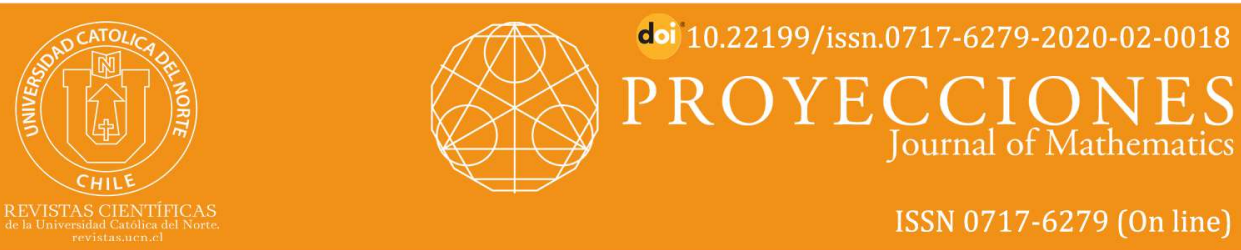

\title{
Computing the metric dimension of Kayak Paddles graph and Cycles with chord
}

\author{
Ali Ahmad ${ }^{1}$ (1) orcid.org/0000-0003-3434-9908 \\ Martin Bača ${ }^{2}$ (1) orcid.org/0000-0002-5758-0347 \\ Saba Sultan ${ }^{3}$ \\ ${ }^{1}$ Jazan University, College of Computer Science \& Information Systems, Jazan, Saudi Arabia. \\ ahmadsms@gmail.com \\ ${ }^{2}$ Technical University, Dept.of Applied Mathematics and Informatics, Košice, Slovakia. \\ martin.baca@tuke.sk \\ ${ }^{3}$ Government College University, Abdus Salam School of Mathematical Sciences, Lahore, Pakistan. \\ 、sabasultan09@gmail.com
}

Received: March 2019 | Accepted: May 2019

\section{Abstract:}

A set of vertices $W$ is a resolving set of a graph $G$ if every two vertices of $G$ have distinct representations of distances with respect to the set $W$. The number of vertices in a smallest resolving set is called the metric dimension. This invariant has extensive applications in robotics, since the metric dimension can represent the mínimum number of landmarks, which uniquely determine the position of a robot moving in a graph space. Finding the metric dimension of a graph is an NP-hard problem. We present exact values of the metric dimensión of Kayak Paddles graph and Cycles with chord.

Keywords: Metric dimension; Resolving set; Kayak paddles; Robotics.

MSC (2010): 05C12; 05C35; 68R10.

\section{Cite this article as (IEEE citation style)}

A. Ahmad, M. Bača, and S. Sultan, "Computing the metric dimen-

sion of Kayak Paddles graph and Cycles with chord", Proyecciones (Antofagasta, On line), vol. 39, no. 2, pp. 287-300, Apr. 2020, doi: 10.22199/issn.0717-6279-2020-02-0018.

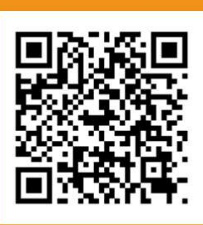

Article copyright: (C) 2020 Ali Ahmad, Martin Bača and Saba Sultan. This is an open access article distributed under the terms of the Creative Commons Licence, which permits unrestricted use and distribution provided the original author and source are credited.

(cc) BY 


\section{Introduction}

Consider the following problem, which was studied in [19]. A robot moves in a space, which is modeled by a graph. The robot moves from a node to a node, and it can locate itself by the presence of distinctively labeled landmark nodes. The position of robot is represented by its distances to a set of landmarks. The problem is to find the minimum number of landmarks required, and to find out where they should be placed, such that the robot can always determine its location. In graph theory language, a minimum set of landmarks which uniquely determine the position of robot is called a metric basis, and the minimum number of landmarks is called the metric dimension.

Slater $[24,25]$ introduced the concept of metric dimension and studied independently by Harary and Melter [8]. Slater referred to a metric dimension of a graph as its location number and motivated the study of this invariant by its application to the placement of minimum number of loran/sonar detecting devices in a network so that the position of every vertex in the network can be uniquely represented in terms of its distances to the devices in the set. Applications of the study of metric dimension to the problem of pattern recognition and image processing are given in [20].

Let $G$ be a connected graph with the vertex set $V(G)$. The distance $d(u, v)$ between two vertices $u, v \in V(G)$ is the number of edges in a shortest path between them. A vertex $w$ resolves a pair of vertices $u, v$ if $d(u, w) \neq d(v, w)$. For an ordered set of vertices $W=\left\{w_{1}, w_{2}, \ldots, w_{z}\right\}$, the representation of distances of a vertex $v$ with respect to $W$ is the ordered $z$-tuple

$$
r(v \mid W)=\left(d\left(v, w_{1}\right), d\left(v, w_{2}\right), \ldots, d\left(v, w_{z}\right)\right) .
$$

A set of vertices $W \subset V(G)$ is a resolving set of $G$ if every two vertices of $G$ have distinct representations (if every pair of vertices of $G$ is resolved by some vertex of $W$ ). The cardinality of a smallest resolving set is called the metric dimension and it is denoted by $\operatorname{dim}(G)$. Note that the $i$-th coordinate in $r(v \mid W)$ is 0 if and only if $v=w_{i}$. This means that in order to show that $W$ is a resolving set of $G$, it suffices to verify that $r(u \mid W) \neq$ $r(v \mid W)$ for every pair of distinct vertices $u, v \in V(G) \backslash W$.

The metric dimension of various classes of graphs has been investigated for four decades. From [7] it follows that the question whether the metric dimension of a graph is less than a given value, is an NP-complete problem. In [17] and [22] the authors considered the metric dimension of the 
lexicographic product of graphs and results on the metric dimension of the corona product of graphs were presented by Iswadi, Baskoro and Simanjuntak [16]. Cáceres et. al. [5] studied the Cartesian product $G \square H$ of two graphs $G$ and $H$. They proved that for all $m, n \geq 3$,

$$
\operatorname{dim}\left(C_{m} \square C_{n}\right)= \begin{cases}3, & \text { if } m \text { or } n \text { is odd } \\ 4, & \text { otherwise, }\end{cases}
$$

and for all $m \geq 2$ and $n \geq 3$, we have

$$
\operatorname{dim}\left(P_{m} \square C_{n}\right)= \begin{cases}2, & \text { if } n \text { is odd } \\ 3, & \text { if } n \text { is even, }\end{cases}
$$

where $C_{n}$ is the cycle of order $n$ and $P_{m}$ is the path of order $m$. Naeem and Imran [21] used a different technique to prove this result and they also studied the metric dimension of the Cartesian product of the path and the square of the cycle. They showed that for $n \geq 5$,

$$
\operatorname{dim}\left(P_{m} \square C_{n}^{2}\right)= \begin{cases}3, & \text { if } n \equiv 0,2,3(\bmod 4) \\ \leq 4, & \text { otherwise. }\end{cases}
$$

To find the metric dimension of different families of graphs has been a continuous subject of interest for researchers. The metric dimension of Cayley digraphs has been obtained in [6]. Metric dimension of some chemical graphs (nanotubes) has been found recently in [23]. The metric dimension of unicyclic graphs, regular bipartite graphs, Jahangir graph, Möbius ladder and circulant graphs has been discussed in [26], [3], [27], [1] and [14] respectively. Examples of families of graphs with constant metric dimension can be found in $[9,10,11,12,13,15,18]$.

In this paper, we solve the problem of metric dimension for kayak paddles graph and cycles with chord. We proved that both families possess the constant metric dimension 2 .

\section{Metric dimension of Kayak Paddles graph}

A kayak paddle is a graph made of two cycles joined by a path. We can define $K P(l, m, n)$ as two cycles of length $l$ and $m$ joined by a path of length $n$. For $l \geq 3, m \geq 3, n \geq 2$, we have $V(K P(l, m, n))=\left\{u_{1}, u_{2}, \ldots, u_{l}, v_{1}, v_{2}, \ldots\right.$, $\left.v_{m}, w_{1}, w_{2}, \ldots, w_{n-1}\right\}$ and $E(K P(l, m, n))=\left\{u_{i} u_{i+1}: 1 \leq i \leq l\right\} \cup\left\{v_{i} v_{i+1}:\right.$ $1 \leq i \leq m\} \cup\left\{w_{i} w_{i+1}: 1 \leq i \leq n-2\right\} \cup\left\{u_{1} w_{1}, w_{n-1} v_{1}\right\}$, where $u_{l+1}=u_{1}$ and $v_{m+1}=v_{1}$. As a convention, we will label the vertices of $C_{l}$ in counter 
clockwise direction and vertices of $C_{m}$ in clockwise direction. The graph $K P(8,7,5)$ is shown in Figure 1.

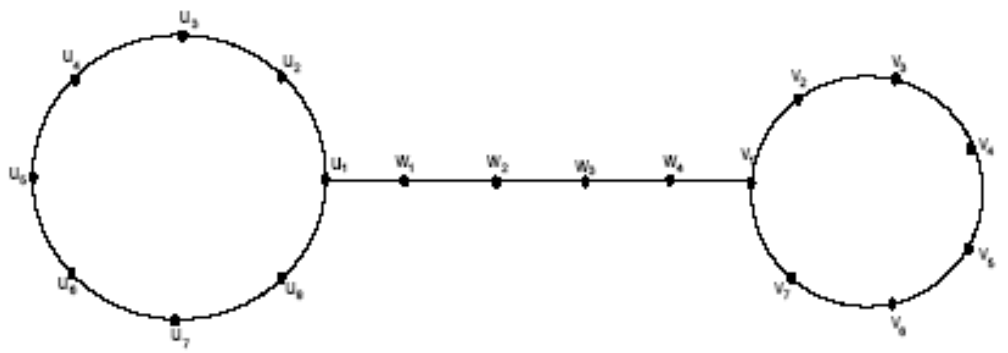

Figure 1: The graph $K P(8,7,5)$

Theorem 1. Let $G=K P(l, m, n)$, then $\operatorname{dim}(G)=2$.

Proof. As metric dimension of a graph is 1 if and only if it is a path [4], this proves the fact that

$$
\operatorname{dim}(G) \geq 2 .
$$

For reverse inequality, we will prove the existence of a resolving set of cardinality 2. There are three different array, which need to be discussed here.

\section{Case 1: When $l$ and $m$ both are even.}

Suppose $l=2 p$ and $m=2 q$, where $p, q \geq 2$. Consider the set $W=\left\{u_{p}, v_{q}\right\}$, then the representation of each vertex with respect to $W$ is given by:

$$
\begin{aligned}
& r\left(u_{i} \mid W\right)=\left\{\begin{array}{l}
(p-i, q+n-2+i) \quad \text { for } \quad 1 \leq i \leq p \\
(i-p, 2 p+q+n-i) \quad \text { for } \quad p+1 \leq i \leq l
\end{array}\right. \\
& r\left(u_{i} \mid W\right)=\left\{\begin{array}{l}
(p+n-2+i, q-i) \quad \text { for } \quad 1 \leq i \leq q \\
(p+2 q+n-i, i-q) \quad \text { for } \quad q+1 \leq i \leq m .
\end{array}\right. \\
& r\left(w_{i} \mid W\right)=(p-1+i, q+n-1-i) \quad \text { for } 1 \leq i \leq n-1 .
\end{aligned}
$$

Case 2: When $l$ is odd and $m$ is even. 
In this case, we have the following subcases:

Subcase I: $l=3$ and $m=2 q, q \geq 2$

Consider the set $W=\left\{u_{2}, v_{q}\right\}$, then the representation of each vertex with respect to $W$ is given by:

$$
\begin{gathered}
r\left(u_{i} \mid W\right)=\left\{\begin{array}{c}
(2-i, q+n-2+i) \text { for } 1 \leq i \leq 2 ; \\
(1, q+n) \text { for } i=3 .
\end{array}\right. \\
r\left(v_{i} \mid W\right)=\left\{\begin{array}{l}
(n+i, q-i) \text { for } 1 \leq i \leq q ; \\
(2 q+n+2-i, i-q) \text { for } q+1 \leq i \leq m .
\end{array}\right. \\
r\left(w_{i} \mid W\right)=(1+i, q+n-1-i) \text { for } 1 \leq i \leq n-1 .
\end{gathered}
$$

Subcase II: $l=5$ and $m=2 q, q \geq 2$ Consider the set $W=\left\{u_{2}, v_{q}\right\}$, then the representation of each vertex with respect to $W$ is given by:

$$
\begin{gathered}
r\left(u_{i} \mid W\right)=\left\{\begin{array}{l}
(2-i, q+n-2+i) \text { for } 1 \leq i \leq 2 ; \\
(i-2, q+n+1) \text { for } 3 \leq i \leq 4 ; \\
(2, q+n) \text { for } i=5 .
\end{array}\right. \\
r\left(v_{i} \mid W\right)=\left\{\begin{array}{l}
(n+i, q-i) \text { for } 1 \leq i \leq q ; \\
(2 q+n+2-i, i-q) \text { for } q+1 \leq i \leq m .
\end{array}\right. \\
r\left(w_{i} \mid W\right)=(1+i, q+n-1-i) \quad, 1 \leq i \leq n-1 .
\end{gathered}
$$

Subcase III: $l=7$ and $m=2 q, q \geq 2$

Consider the set $W=\left\{u_{3}, v_{q}\right\}$, then the representation of each vertex with respect to $W$ is given by:

$$
\begin{gathered}
r\left(u_{i} \mid W\right)=\left\{\begin{array}{l}
(3-i, q+n-2+i) \text { for } 1 \leq i \leq 3 ; \\
(i-3, q+n+2) \text { for } 4 \leq i \leq 5 ; \\
(3, q+n+7-i) \text { for } 6 \leq i \leq 7 .
\end{array}\right. \\
r\left(v_{i} \mid W\right)=\left\{\begin{array}{l}
(n+1+i, q-i) \text { for } 1 \leq i \leq q ; \\
(2 q+n+3-i, i-q) \text { for } q+1 \leq i \leq m .
\end{array}\right. \\
r\left(w_{i} \mid W\right)=(2+i, q+n-1-i) \text { for } 1 \leq i \leq n-1 .
\end{gathered}
$$


Subcase IV: $l=2 p+1, p \geq 4$ and $m=2 q, q \geq 2$

Consider the set $W=\left\{u_{p}, v_{q}\right\}$, then the representation of each vertex with respect to $W$ is given by:

$$
\begin{gathered}
r\left(u_{i} \mid W\right)=\left\{\begin{array}{l}
(p-i, q+n-2+i) \quad \text { for } 1 \leq i \leq p ; \\
(i-p, p+q+n-1) \text { for } \\
p+1 \leq i \leq p+2 ; \\
(i-p, 2 p+q+n+1-i) \quad \text { for } p+3 \leq i \leq 2 p-1 \\
(p, 2 p+q+n+1-i) \text { for } 2 p \leq i \leq l .
\end{array}\right. \\
r\left(v_{i} \mid W\right)= \begin{cases}(p+n-2+i, q-i) & \text { for } 1 \leq i \leq q ; \\
(p+2 q+n-i, i-q) & \text { for } q+1 \leq i \leq m .\end{cases} \\
r\left(w_{i} \mid W\right)=(p-1+i, q+n-1-i) \text { for } 1 \leq i \leq n-1 .
\end{gathered}
$$

\section{Case 3: When $l$ and $m$ both are odd.}

In this case, we have the following subcases:

Subcase I: $l=3$ and $m=2 q+1, q \geq 1$ There are four possibilities, which must be consider here.

(i): If $m=3$. Consider the set $W=\left\{u_{2}, v_{2}\right\}$, then the representation of each vertex with respect to $W$ is given by:

$$
\begin{gathered}
r\left(u_{i} \mid W\right)=\left\{\begin{array}{l}
(2-i, n+i) \text { for } 1 \leq i \leq 2 ; \\
(1, n+2) \text { for } i=3 .
\end{array}\right. \\
r\left(v_{i} \mid W\right)=\left\{\begin{array}{l}
(n+i, 2-i) \text { for } 1 \leq i \leq 2 ; \\
(n+2,1) \text { for } i=3 .
\end{array}\right. \\
r\left(w_{i} \mid W\right)=(1+i, n+1-i) \text { for } 1 \leq i \leq n-1 .
\end{gathered}
$$

(ii): If $m=5$. Consider the set $W=\left\{u_{2}, v_{2}\right\}$, then the representation of each vertex with respect to $W$ is given by:

$$
r\left(u_{i} \mid W\right)=\left\{\begin{array}{l}
(2-i, n+i) \quad \text { for } 1 \leq i \leq 2 \\
(1, n+2) \quad \text { for } i=3
\end{array}\right.
$$




$$
\begin{gathered}
r\left(v_{i} \mid W\right)=\left\{\begin{array}{c}
(n+i, 2-i) \quad \text { for } 1 \leq i \leq 2 ; \\
(n+3, i-2) \text { for } 3 \leq i \leq 4 ; \\
(n+2,2) \text { for } i=5 .
\end{array}\right. \\
r\left(w_{i} \mid W\right)=(1+i, n+1-i) \text { for } 1 \leq i \leq n-1 .
\end{gathered}
$$

(iii): If $m=7$. Consider the set $W=\left\{u_{2}, v_{3}\right\}$, then the representation of each vertex with respect to $W$ is given by:

$$
\begin{gathered}
r\left(u_{i} \mid W\right)= \begin{cases}(2-i, n+1+i) \quad \text { for } 1 \leq i \leq 2 ; \\
(1, n+3) \text { for } i=3 .\end{cases} \\
r\left(v_{i} \mid W\right)= \begin{cases}(n+i, 3-i) & \text { for } 1 \leq i \leq 3 ; \\
(n+4, i-3) & \text { for } 4 \leq i \leq 5 ; \\
(n+9-i, 3) & \text { for } 6 \leq i \leq 7 .\end{cases} \\
r\left(w_{i} \mid W\right)=(1+i, n+2-i) \text { for } 1 \leq i \leq n-1 .
\end{gathered}
$$

(iv): If $m=2 q+1, q \geq 4$. Consider the set $W=\left\{u_{2}, v_{q}\right\}$, then the representation of each vertex with respect to $W$ is given by:

$$
\begin{gathered}
r\left(u_{i} \mid W\right)=\left\{\begin{array}{l}
(2-i, q+n-2+i) \quad \text { for } 1 \leq i \leq 2 \\
(1, q+n) \text { for } i=3
\end{array}\right. \\
r\left(v_{i} \mid W\right)=\left\{\begin{array}{l}
(n+i, q-i) \text { for } 1 \leq i \leq q ; \\
(q+n+1, i-q) \text { for } q+1 \leq i \leq q+2 \\
(2 q+n+3-i, i-q) \text { for } q+3 \leq i \leq 2 q-1 \\
(2 q+n+3-i, q) \text { for } 2 q \leq i \leq m
\end{array}\right. \\
r\left(w_{i} \mid W\right)=(1+i, q+n-1-i) \text { for } 1 \leq i \leq n-1 .
\end{gathered}
$$

Subcase II: $l=5$ and $m=2 q+1, q \geq 2$

There are three possibilities, which must be consider here.

(i): If $m=5$. Consider the set $W=\left\{u_{2}, v_{2}\right\}$, then the representation of each vertex with respect to $W$ is given by:

$$
r\left(u_{i} \mid W\right)=\left\{\begin{array}{rc}
2-i, n+i) & \text { for } 1 \leq i \leq 2 \\
(i-2, n+3) & \text { for } 3 \leq i \leq 4 \\
(2, n+2) & \text { for } i=5
\end{array}\right.
$$




$$
\begin{gathered}
r\left(v_{i} \mid W\right)=\left\{\begin{array}{c}
(n+i, 2-i) \quad \text { for } 1 \leq i \leq 2 ; \\
(n+3, i-2) \quad \text { for } 3 \leq i \leq 4 \\
(n+2,2) \text { for } i=5 .
\end{array}\right. \\
r\left(w_{i} \mid W\right)=(1+i, n+1-i) \quad \text { for } 1 \leq i \leq n-1 .
\end{gathered}
$$

(ii): If $m=7$. Consider the set $W=\left\{u_{2}, v_{3}\right\}$, then the representation of each vertex with respect to $W$ is given by:

$$
\begin{gathered}
r\left(u_{i} \mid W\right)=\left\{\begin{array}{l}
(2-i, n+1+i) \text { for } 1 \leq i \leq 2 \\
(i-2, n+4) \text { for } 3 \leq i \leq 4 ; \\
(2, n+3) \text { for } i=5
\end{array}\right. \\
r\left(v_{i} \mid W\right)= \begin{cases}(n+i, 3-i) & \text { for } 1 \leq i \leq 3 \\
(n+4, i-3) & \text { for } 4 \leq i \leq 5 \\
(n+9-i, 3) & \text { for } 6 \leq i \leq 7\end{cases}
\end{gathered}
$$

(iii): If $m=2 q+1, q \geq 4$. Consider the set $W=\left\{u_{2}, v_{q}\right\}$, then the representation of each vertex with respect to $W$ is given by:

$$
\begin{gathered}
r\left(u_{i} \mid W\right)=\left\{\begin{array}{l}
(2-i, q+n-2+i) \text { for } 1 \leq i \leq 2 ; \\
(i-2, q+n+1) \text { for } 3 \leq i \leq 4 ; \\
(2, q+n) \text { for } i=5 .
\end{array}\right. \\
r\left(v_{i} \mid W\right)=\left\{\begin{array}{l}
(n+i, q-i) \text { for } 1 \leq i \leq q ; \\
(q+n+1, i-q) \text { for } q+1 \leq i \leq q+2 ; \\
(2 q+n+3-i, i-q) \text { for } q+3 \leq i \leq 2 q-1 ; \\
(2 q+n+3-i, q) \text { for } 2 q \leq i \leq m .
\end{array}\right. \\
r\left(w_{i} \mid B\right)=(1+i, q+n-1-i) \text { for } 1 \leq i \leq n-1 .
\end{gathered}
$$

Subcase III: $l=7$ and $m=2 q+1, q \geq 3$

There are two possibilities, which must be consider here.

(i): If $m=7$. Consider the set $W=\left\{u_{3}, v_{3}\right\}$, then the representation of each vertex with respect to $W$ is given by:

$$
r\left(u_{i} \mid W\right)=\left\{\begin{array}{cc}
3-i, n+1+i) & \text { for } 1 \leq i \leq 3 \\
(i-3, n+5) & \text { for } 4 \leq i \leq 5 \\
(3, n+10-i) & \text { for } 6 \leq i \leq 7
\end{array}\right.
$$




$$
\begin{gathered}
r\left(v_{i} \mid W\right)=\left\{\begin{array}{l}
(n+1+i, 3-i) \\
\text { for } 1 \leq i \leq 3 \\
(n+5, i-3) \\
\text { for } 4 \leq i \leq 5 ; \\
(n+10-i, 3) \\
\text { for } 6 \leq i \leq 7 .
\end{array}\right. \\
r\left(w_{i} \mid W\right)=(2+i, n+2-i) \text { for } 1 \leq i \leq n-1 .
\end{gathered}
$$

(ii): If $m=2 q+1, q \geq 4$. Consider the set $W=\left\{u_{3}, v_{q}\right\}$, then the representation of each vertex with respect to $W$ is given by:

$$
\begin{gathered}
r\left(u_{i} \mid W\right)=\left\{\begin{array}{l}
(3-i, q+n-2+i) \text { for } 1 \leq i \leq 3 \\
(i-3, q+n+2) \text { for } 4 \leq i \leq 5 \\
(3, q+n+7-i) \text { for } 6 \leq i \leq 7
\end{array}\right. \\
r\left(v_{i} \mid B\right)=\left\{\begin{array}{l}
(n+1+i, q-i) \text { for } 1 \leq i \leq q \\
(q+n+2, i-q) \text { for } q+1 \leq i \leq q+2 \\
(2 q+n+4-i, i-q) \text { for } q+3 \leq i \leq 2 q-1 \\
(2 q+n+4-i, q) \text { for } 2 q \leq i \leq m
\end{array}\right. \\
r\left(w_{i} \mid B\right)=(2+i, q+n-1-i) \text { for } 1 \leq i \leq n-1 .
\end{gathered}
$$

Subcase IV: $l=2 p+1, p \geq 4$ and $m=2 q+1, q \geq 4$

Consider the set $W=\left\{u_{p}, v_{q}\right\}$, then the representation of each vertex with respect to $W$ is given by:

$$
\begin{aligned}
& r\left(u_{i} \mid W\right)=\left\{\begin{array}{l}
(p-i, q+n-2+i) \quad \text { for } 1 \leq i \leq p \\
(i-p, p+q+n-1) \text { for } p+1 \leq i \leq p+2 \\
(i-p, 2 p+q+n+1-i) \text { for } p+3 \leq i \leq 2 p-1 \\
(p, 2 p+q+n+1-i) \text { for } 2 p \leq i \leq l
\end{array}\right. \\
& r\left(v_{i} \mid W\right)=\begin{array}{l}
(p+n-2+i, q-i) \quad \text { for } 1 \leq i \leq q \\
(p+q+n-1, i-q) \text { for } q+1 \leq i \leq q+2 \\
(p+2 q+n+1-i, i-q) \text { for } q+3 \leq i \leq 2 q-1 \\
(p+2 q+n+1-i, q) \text { for } 2 q \leq i \leq m
\end{array} \\
& r\left(w_{i} \mid W\right)=(p-1+i, q+n-1-i) \quad \text { for } 1 \leq i \leq n-1 .
\end{aligned}
$$


It can be seen that, for every case, each vertex has unique representation with respect to $W$, which proves $W$ to be resolving set and hence

$$
\operatorname{dim}(G) \leq 2
$$

Now from inequalities (2.1) and (2.2), it can be concluded that metric dimension of $K P(l, m, n)=\operatorname{dim}(G)=2$.

Remark: Note that $\operatorname{KP}(1, \mathrm{~m}, 1)$ has also metric dimension 2. In this case, $V(K P(l, m, 1))=\left\{u_{1}, u_{2}, \ldots, u_{l}, v_{1}, v_{2}, \ldots, v_{m}\right\}$ and $E(K P(l, m, 1))=\left\{u_{i} u_{i+1}\right.$ : $1 \leq i \leq l\} \cup\left\{v_{i} v_{i+1}: 1 \leq i \leq m\right\} \cup\left\{u_{1} v_{1}\right\}$, where $u_{l+1}=u_{1}$ and $v_{m+1}=v_{1}$. The resolving set in each case is the same as discussed above, however for representations of vertices with respect to resolving set, eliminate $r\left(w_{i} \mid W\right)$ completely and put $n=1$ in $r\left(u_{i} \mid W\right)$ and $r\left(v_{i} \mid W\right)$.

\section{Metric dimension of Cycles with chord}

Let us denote the graph constructed from a cycle $C_{n}$ by joining two vertices whose distance in the cycle is $t$, by $C_{n}^{t}$. For $n \geq 4,2 \leq t \leq n-2$, the graph $C_{n}^{t}$ is of course also the graph $C_{n}^{n-t}$. Therefore, to find the metric dimension, it suffices to consider $t \leq\left[\frac{n}{2}\right]$ for given value of $n$. Let $V\left(C_{n}^{t}\right)=\left\{u_{1}, u_{2}, \ldots, u_{n}\right\}$ and $E\left(C_{n}^{t}\right)=\left\{u_{i} u_{i+1}: 1 \leq i \leq n\right\} \cup\left\{u_{1} u_{t+1}\right\}$ where $u_{n+1}=u_{1}$. As a convention, we will label the vertices in clockwise direction. For example $C_{14}^{5}$ is illustrated in Figure 2.

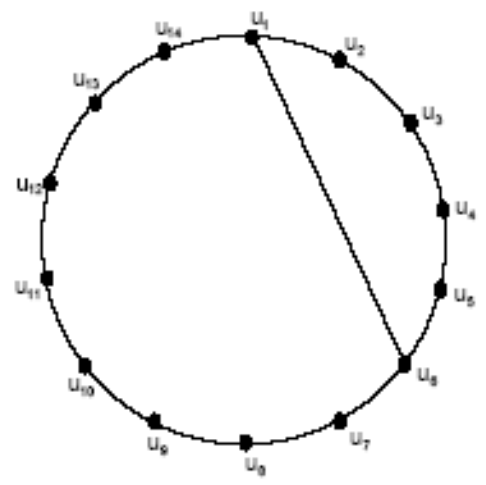

Figure 2: The graph $C_{14}^{5}$

Theorem 2. Let $G=C_{n}^{t}$, then $\operatorname{dim}(G)=2$. 
Proof. Clearly we have

$$
\operatorname{dim}(G) \geq 2
$$

since metric dimension of a graph is 1 if and only if it is a path [4]. For reverse inequality, we will show that there exist a resolving set of cardinality 2. For this, the following array need to be discussed.

\section{Case 1: When $n$ and $t$ both are even.}

Suppose $n=2 k, t=2 p$. Consider the set $W=\left\{u_{k+p}, u_{k+p+1}\right\}$. The representation of each vertex $u_{i}$ with respect to $W$ is given by:

$$
r\left(u_{i} \mid W\right)=\left\{\begin{array}{c}
k-p-1+i, k-p-1+i) \quad \text { for } 1 \leq i \leq p \\
(k+p-i, k+p+1-i) \quad \text { for } p+1 \leq i \leq k+p \\
(i-k-p, i-k-p-1) \text { for } k+p+1 \leq i \leq n
\end{array}\right.
$$

\section{Case 2: When $n$ is odd and $t$ is even.}

Suppose $n=2 k+1, t=2 p$. Consider the set $W=\left\{u_{k+p+1}, u_{k+p+2}\right\}$. The representation of each $u_{i}$ with respect to $W$ is given by:

$$
r\left(u_{i} \mid W\right)= \begin{cases}(k-p+i, k-p-1+i) \quad \text { for } 1 \leq i \leq p \\ (k, k) \text { for } i=p+1 ; & \\ (k+p+1-i, k+p+2-i) & \text { for } p+2 \leq i \leq k+p+1 \\ (i-k-p-1, i-k-p-2) & \text { for } k+p+2 \leq i \leq n\end{cases}
$$

\section{Case 3: When $n$ is even and $t$ is odd.}

Suppose $n=2 k, t=2 p+1$. Consider the set $W=\left\{u_{k+p+1}, u_{k+p+2}\right\}$. The representation of each vertex $u_{i}$ with respect to $W$ is given by:

$$
r\left(u_{i} \mid W\right)= \begin{cases}(k-p-1+i, k-p-2+i) & \text { for } 1 \leq i \leq p+1 \\ (k+p+1-i, k+p+2-i) & \text { for } p+2 \leq i \leq k+p+1 \\ (i-k-p-1, i-k-p-2) & \text { for } k+p+2 \leq i \leq n\end{cases}
$$

\section{Case 4: When $n$ and $t$ both are odd.}

Suppose $n=2 k+1, t=2 p+1$. Consider the set $W=\left\{u_{k+p+1}, u_{k+p+2}\right\}$. The representation of each vertex $u_{i}$ with respect to $W$ is given by: 


$$
r\left(u_{i} \mid W\right)= \begin{cases}(k-p-1+i, k-p-1+i) & \text { for } 1 \leq i \leq p+1 \\ (k+p+1-i, k+p+2-i) & \text { for } p+2 \leq i \leq k+p+1 \\ (i-k-p-1, i-k-p-2) & \text { for } k+p+2 \leq i \leq n\end{cases}
$$

It can be seen that, for every case, each $u_{i}$ has unique representation with respect to $W$, which proves $W$ to be resolving set and hence

$$
\operatorname{dim}(G) \leq 2 .
$$

By comparing inequalities (3.1) and (3.2), it can be seen that metric dimension of $C_{n}^{t}=\operatorname{dim}(G)=2$.

\section{Conclusion}

The metric dimension for the graph $K P(n, n, 1), n \geq 3$ has been discussed in [2]. Thus, we have found the metric dimension of $\operatorname{KP}(1, m, n)$ in more general frame. The family $C_{n}^{t}$ is also interesting, as it is similar to a circle but distance between vertices of circle get change by adding one extra edge here. Important phenomena is that there is no change in metric dimension i.e. $\operatorname{dim}\left(C_{n}\right)=\operatorname{dim}\left(C_{n}^{t}\right)$. In this way, our work in this direction naturally arise a question of characterization of changes in graph which could change the metric dimension of that graph by adding just one extra edge.

\section{References}

[1] M. Ali, G. Ali, A. Q. Baig, and M. K. Shafiq, "On the metric dimension of Möbius ladders", Ars combinatoria, vol. 105, pp. 403-410, Jul. 2012.

[2] M. Ali, G. Ali, U. Ali, and M. T. Rahim, "On cycle related graphs with constant metric dimension", Open journal of discrete mathematics, vol. 02, no. 01, pp. 21-23, 2012, doi: $10.4236 /$ ojdm.2012.21005.

[3] M. Bača, E. T. Baskoro, A. N. M. Salman, S. W. Saputro, and D.Suprijanto, "The metric dimension of regular bipartite graphs", Bulletin mathématique de la Société des Sciences Mathématiques de Roumanie, vol. 54, no. 1, pp. 15-28, 2011. [On line]. Available: https://bit.ly/2S12YpQ

[4] C. Hernando, M. Mora, I. M. Pelayo, C. Seara, J. Cáceres, and M. L. Puertas, "On the metric dimension of some families of graphs", Electronic notes in discrete mathematics, vol. 22, pp. 129-133, Oct. 2005, doi: 10.1016/j.endm.2005.06.023.

[5] J. Cáceres, C. Hernando, M. Mora, I. M. Pelayo, M. L. Puertas, C. Seara, and D. R. Wood, "On the metric dimension of cartesian products of graphs", SIAM journal on discrete mathematics, vol. 21, no. 2, pp. 423-441, 2007, doi:10.1137/050641867. 
[6] M. Fehr, S. Gosselin, and O. R. Oellermann, "The metric dimension of Cayley digraphs", Discrete mathematics, vol. 306, no. 1, pp. 31-41, Jan. 2006, doi: 10.1016/j.disc.2005.09.015.

[7] M. R. Garey and D. S. Johnson, Computers and intractability: a guide to the theory of NP - completeness. San Francisco , CA: W.H. Freeman and Co., 1979.

[8] F. Harary and R. A. Melter, "On the metric dimension of a graph", Ars combinatoria, vol. 2, pp. 191-195, 1976.

[9] M. Imran, A. Q. Baig, and A. Ahmad, "Families of plane graphs with constant metric dimensión", Utilitas mathematica, in press.

[10] M. Imran, A. Q. Baig, M. K. Shafiq and A. Semeničová, "Classes of convex polytopes with constant metric dimensión", Utilitas mathematica, in press.

[11] M. Imran, S. A. Bokhary, and A. Q. Baig, "On families of convex polytopes with constant metric dimension", Computers \& mathematics with applications, vol. 60, no. 9, pp. 2629-2638, Nov. 2010, doi: 10.1016/j.camwa.2010.08.090

[12] M. Imran and A. Q. Baig, "A special class of convex polytopes with constant metric dimension", Journal of combinatorial mathematics and combinatorial computing, vol. 77, pp. 197-205, May 2011.

[13] M. Imran and A. Q. Baig, "An infinite class of polytopes with constant metric dimension", Journal of combinatorial mathematics and combinatorial computing, in press.

[14] M. Imran, A. Baig, S. A. Bokhary, and I. Javaid, "On the metric dimension of circulant graphs", Applied mathematics letters, vol. 25, no. 3, pp. 320-325, Mar. 2012, doi: 10.1016/j.aml.2011.09.008.

[15] M. Imran, A. Q. Baig, S. A. Bokhary, and E.T. Baskoro, "New classes of convex polytopes with constant metric dimension", Utilitas mathematica, in press.

[16] H. Iswadi, E. T. Baskoro, and R. Simanjuntak, "On the metric dimension of corona product of graphs", Far east journal of mathematical sciences, vol. 52, no. 2, pp. 155-170, May 2011. [On line]. Available: https://bit.ly/3bCnuF2

[17] M. Jannesari and B. Omoomi, "The metric dimension of the lexicographic product of graphs", Discrete mathematics, vol. 312, no. 22, pp. 3349-3356, Nov. 2012, doi: 10.1016/j.disc.2012.07.025.

[18] I. Javaid, M. T. Rahim, and K. Ali, "Families of regular graphs with constant metric dimensión", Utilitas mathematica, vol. 75, pp. 21-33, 2008.

[19] S. Khuller, B. Raghavachari, and A. Rosenfeld, "Landmarks in graphs", Discrete applied mathematics, vol. 70, no. 3, pp. 217-229, Oct. 1996, doi: 10.1016/0166-218x(95)00106-2.

[20] R. A. Melter and I. Tomescu, "Metric bases in digital geometry", Computer vision, graphics, and image processing, vol. 25, no. 1, pp. 113-121, Jan. 1984, doi: 10.1016/0734-189X(84)90051-3.

[21] R. Naeem and M. Imran, "Metric dimension and exchange property for resolving sets in rotationally-symmetric graphs", Applied Mathematics \& Information Sciences, vol. 8, no. 4, pp. 1665-1674, Jul. 2014, doi: 10.12785/amis/080422.

[22] S. Saputro, R. Simanjuntak, S. Uttunggadewa, H. Assiyatun, E. Baskoro, A. Salman, and M. Bača, "The metric dimension of the lexicographic product of graphs", Discrete mathematics, vol. 313, no. 9, pp. 1045-1051, May 2013, doi: 10.1016/j.disc.2013.01.021.

[23] H. M. A. Siddiqui and M. Imran, "Computation of metric dimension and partition dimension of nanotubes", Journal of computational and theoretical nanoscience, vol. 12, no. 2, pp. 199-203, Feb. 2015, doi: 10.1166/jctn.2015.3717.

[24] P. J. Slater, "Dominating and reference sets in graphs", Journal of mathematical and physical sciences, vol. 22, n. 4. pp. 445-455, 1998.

[25] P. J. Slater, "Leaves of tres", Congressus numerantium, vol. 14, pp. 549-559, 1975. 
[26] C. Poisson and P. Zhang, "The metric dimension of unicyclic graphs", Journal of combinatorial mathematics and combinatorial computing, vol. 40 , pp. 17-32, 2002.

[27] I. Tomescu and I. Javaid, "On the metric dimension of Jahangir graph", Bulletin mathématique de la Société des Sciences Mathématiques de Roumanie, vol. 50, no. 4, pp. 371-376, 2007. [On line]. Available: https://bit.ly/2VVx4Mq 